mais intensos de epistaxe, é necessária a transfusão de plaquetas por plasmaferese. Conclusão: a Síndrome de Bernard-Soulier, apesar de rara, deve fazer parte da investigação diagnóstica nos casos epistaxes recorrentes, principalmente quando intensas e com início na infância.

\section{SÍNDROME DE VAN DER HOEVE E KLEYN: RELATO DE CASO}

Appolonio F, Aragão MC, Furlan M, Rapoport PB, Yoshimura R, Zanini RVR

raulzani@ajato.com.br

Objetivo: A proposta dos autores é relatar o caso de um paciente portador da síndrome de Van der Hoeve e Kleyn, seguido de um breve levantamento bibliográfico sobre esta rara doença. Relato de Caso: M.C.N.A., 60 anos, sexo feminino, branca, com queixa de zumbido constante na orelha esquerda, tipo "chiado", iniciado há 10 anos, acompanhado de hipoacusia progressiva ipsilateral. Ao exame físico chamava atenção o aspecto azulado de suas escleras. Interrogada sobre antecedentes mórbidos a paciente referiu múltiplas fraturas desde a adolescência. A otoscopia estava normal. A audiometria tonal demonstrou disacusia condutiva e curva timpanométrica $A r$ em orelha direita. A hipótese diagnóstica foi osteogênese imperfeita com acometimento otológico: síndrome de Van der Hoeve e Kleyn. Discussão: Em 1918, Van der Hoeve e de Kleyn descreveram o primeiro caso de um paciente que sofria de fraturas espontâneas, possuía esclera azul e perda auditiva. Desde então, tem se dado mais atenção à osteogênese imperfeita, uma doença de transmissão autossômica dominante, caracterizada por um defeito na síntese de colágeno tipo I, que impede a ossificação normal da cortical do osso associado a hipoacusia. A audição é afetada em 20 a $40 \%$ dos portadores, sendo clínica e radiologicamente semelhante a otospongiose, uma patologia também caracterizada por displasia óssea. A estapedotomia apresenta bons resultados na reabilitação audiológica desses pacientes. Conclusão: A otospongiose e a síndrome de Van der Hoeve e Kleyn apresentam características otológicas, clínicas e radiológicas muito semelhantes, sendo necessários mais estudos para esclarecer as relações entre ambas patologias.

\section{UTILIZAÇÃO DA DIETA CETOGÊNICA NO TRATAMENTO DE EPILEPSIA REFRATÁRIA: UMA REVISÃO DA LITERATURA}

\author{
Alessi R, Cvintal v, Felgueira RM, Wajjnsztejn R
}

alessi@uol.com.br

Introdução: A Dieta Cetogênica (DC) é uma dieta com alto teor de gordura, baixo teor de proteína e carboidratos usada no tratamento de epilepsia refratária em crianças, desde a década de 20. Objetivo: Revisar sistematicamente e sintetizar as evidências de resposta à DC na redução das crises epilépticas em crianças com epilepsia refratária, assim como os efeitos colaterais apresentados. Métodos: Trata-se de estudo de revisão da literatura, realizado através de busca na Medline, utilizando-se como palavras-chaves: Dieta Cetogênica e epilepsia refratária. Selecionamos para revisão ensaios clínicos que avaliaram a resposta à dieta e seus efeitos colaterais. Resultados: Os critérios de efetividade foram: 1) completa eliminação das crises epilépticas; 2) >90\% de melhora das crises e 3) Entre $50-90 \%$ de redução das crises. Os principais efeitos colaterais relatados durante o período do jejum foram: desidratação, hipoglicemia, vômitos, diarréia e recusa alimentar. Após este período, as complicações mais freqüentemente observadas foram: nefrolitíase, infecções de repetição, hiperuricemia, hipercalcemia, acidose, hipercolesterolemia, irritabilidade, letargia, neuropatia óptica, recusa alimentar e distúrbios gastrointestinais, como náuseas, vômitos, constipação, diarréia e dor abdominal. Conclusão: As série revisadas suportam que cerca de $50 \%$ das crianças submetidas à DC para tratamento de epilepsia refratária apresentam uma melhora significativa $(>50 \%)$ no controle das crises epilépticas, efetividade esta maior que a de muitas drogas anti-epilépticas de nova geração, com um custo muito menor. Os efeitos colaterais apresentados não foram relevantes a ponto de limitar a aplicação da dieta.

\title{
PÔSTERES EPIDEMIOLÓGICOS
}

\section{A EQUOTERAPIA NA REABILITAÇ̃̃o DE CRIANÇA COM NECESSIDADES HUMANAS BÁSICAS AFETADAS}

Campos TY, Castello GL, Lara SRG

thaisyamasaki@ig.com.br

Introdução: Um terço das doenças infantis acomete o sistema nervoso ou muscular. Muitas doenças são limitadas, predominam ou tem seu início na infância, como a epilepsia, as encefalopatias de origem pré ou perinatal, as afecções musculares, as doenças metabólicas hereditárias, os distúrbios congnitvos e comportamentais entre outros. A identificação, acompanhamento e tratamento de fatores debilitantes da realização de qualquer atividade, devem ser realizados através de programas de reabilitação. Objetivos: Identificar as doenças mais freqüentes entre a população infantil estudada; identificar as melhorias encontradas em crianças com idade de 0 a 10 anos apresentando necessidades humanas básicas afetadas que praticam equoterapia por no mínimo seis meses. Métodos: Foi realizado estudo quantitativo e de levantamento através de questionário aplicado em 14 crianças de 0 a 10 anos que apresentavam necessidades humanas básicas afetadas, que praticam equoterapia por no mínimo seis meses. As variáveis estudadas foram: alimentação, sono, linguagem, aquisição de atividades motoras, atividades da vida diária, socialização e reações emocionais. Resultados: das 14 crianças estudadas, $40 \%$ tinham diagnóstico de encefalopatia crônica não progressiva por anóxia perinatal, $14 \%$ tiveram melhora relacionada à alimentação, $23 \%$ ao sono, $43 \%$ à linguagem, $60 \%$ à AVD, $43 \%$ à socialização e $35 \%$ à atenção. Conclusão: A equoterapia é um programa terapêutico muito efetivo na realização de crianças com necessidades humanas básicas afetadas, podendo-se observar melhoras significativas.

\section{AVALIAÇÃO DO CONTEÚdO DE LIVROS ESCOLARES: VACINAS}

\author{
Succi CM, Succi RCM, Wickbold D
}

daniwick@yahoo.com.br

Introdução: Os livros escolares e os professores do ensino fundamental têm papel importante na divulgação dos conceitos de educação em saúde. Objetivo: Assim, o objetivo deste trabalho foi avaliar o conteúdo desses livros sobre conceitos que trazem sobre vacinação. Métodos: Foram analisados 50 livros escolares da área de Ciências e Biologia, onde os autores verificaram o seu conteúdo na área de vacinação. Resultados: Apenas 3 livros da $8^{a}$ série não continham informações sobre saúde, mas 17 livros $(34 \%)$ não continham qualquer informação sobre vacinação. Dos 33 livros com informações sobre vacinas, 19 (57,5\%) continham informações incorretas, 29/33 (87,8\%) associavam vacina com prevenção de doenças, $10 / 33(30,3 \%)$ citavam o calendário básico de vacinação e apenas $7 / 33(21,2 \%)$ informavam sobre a vacinação de outras faixas etárias que não a pediátrica. As vacinas mais citadas foram: vacina contra a poliomielite (26 citações), contra a tuberculose (23), contra o sarampo (21) e DPT (19). Vacinas contra dengue, febre tifóide, cólera e varíola (7 citações) foram citadas mais vezes que as vacinas contra hepatite A (1), gripe (2), varicela (2) e raiva (3). Alguns dos erros encontrados foram, além da citação de vacinas inexistentes (dengue) ou em desuso (varíola), a associação de vacinas com dor e desconforto, o0 conceito de que vacina é um remédio e a indicação de vacinação apenas para crianças. Conclusão: Os livros escolares perdem a oportunidade de introduzir conceitos corretos e adequados sobre imunizações, na época em que as crianças estão ávidas por novos conhecimentos e podem transmití-los para seus familiares. 
139. CAMPANHAS DE PREVENÇ̃̃O AO CÂNCER DE PELE: PERFIL ESTATÍSTICO DOS PACIENTES DO ABC

Campos VF, Furlan M, Machado Filho CDS, Sá MMS, Schalch FO, Silva CAP

mms_79@terra.com.br

O câncer de pele vem aumentando de forma intensa e alarmante justificando os estudos e as campanhas de prevenção, visando diminuir a morbidade e a mortalidade. Métodos: A liga de Combate ao Câncer de Pele da Faculdade de Medicina do ABC realizou campanhas em parceria com a Sociedade Brasileira de Dermatologia, e por iniciativa própria, entre 2002 a 2002, totalizando 833 pacientes atendidos. Casuística: O levantamento estatístico dos protocolos utilizados demonstrou a procura por pessoas entre 61 e 70 anos $(22,93 \%)$, brancas $(80,31 \%)$ e que se expõem ao sol sem proteção $(61 \%)$, constituindo a faixa de risco de câncer de pele. Das lesões encontradas, $5,52 \%$ eram suspeitas de câncer, das quais $4,56 \%$ correspondem ao carcinoma basocelular, 1,08\% ao carcinoma espinocelular e $0,48 \%$ ao melanoma. As lesões pré-neoplásicas representaram 18,25\% e outras lesões dermatológicas $72,03 \%$. O elevado número dessas últimas deve-se ao despreparo e não conhecimento da população em reconhecer uma lesão suspeita de câncer, levando-as a procurar as campanhas para consulta dermatológica, apesar de não ser o objetivo. Conclusão: Os achados desse estudo comprovam a importância das campanhas. Todos os pacientes foram orientados, através de aulas explicativas, a não se exporem ao sol sem proteção, a fazer o auto exame e alertados quanto aos efeitos acumulativos da exposição ao sol, criando o hábito de prevenção junto à população jovem. Os casos suspeitos de malignidade, foram encaminhados ao seviço de Dermatologia da Faculdade.

\section{CÂNCER GÁSTRICO: UM ESTUDO RETROSPECTIVO DE 985 CASOS NA REGIÃO DO ABC PAULISTA}

Alessi R, Cassefo G, Catapani WR, Chehter EZ, Chicoli FA, Doria PLS alessi@uol.com.br

Introdução: No Brasil, a incidência do câncer gástrico é 28/100.000 no sexo masculino e 15/100.000 no feminino. Na região do ABC Paulista, com 3 milhões de habitantes e alto índice de desenvolvimento (IDH), desconhece-se: 1. incidência, 2. características epidemiológicas, 3. histologia. Dadas as características especiais da região, o comportamento dessa doença seria como em países desenvolvidos ou em desenvolvimento? Métodos: Estudo aberto, seqüencial e retrospectivo (1974-2002) de 985 lâminas de câncer gástrico, analisados pelo Departamento de Patologia da Faculdade de Medicina do ABC. Investigou-se: estadiamento (classificação de Lauren), região gástrica acometida, tipo histológico e classificação de Bormann. Também foram analisadas características epidemiológicas desta população, como sexo, idade e raça. Resultados: Prevalência do sexo masculino $(70,66 \%)$, idade acima de 50 anos $(79,13 \%)$ e raça branca $(79,76 \%)$. O adenocarcinoma presente em $90,86 \%$ das neoplasias e o tipo intestinal representaram $53,22 \%$. Características da neoplasia: diferenciado $-43,63 \%$, infiltrativo $-16,54$, região não-cárdia $-91,52$ e estádio avançado $-94,01 \%$. O teste do Qui-Quadrado demonstrou o adenocarcinoma ser a neoplasia mais comum após os 50 anos $(\mathrm{p}<0,046)$ e outros parâmetros como associação entre idade e tipo, idade e região gástrica, sem diferença estatisticamente significativa. Conclusão: Estes resultados sugerem similaridades entre o ABC Paulista e países desenvolvidos quanto sexo, idade, raça, tipo histológico e região acometida. Entretanto, a alta incidência de câncer em estádio avançado é típica de países em desenvolvimento. Os resultados indicam a necessidade de diagnóstico mais precoce e terapêutica mais eficaz.

\section{DIAGNÓSTICO HISTEROSCÓPICO DE PACIENTES COM SANGRAMENTO UTERINO PÓS-MENOPAUSA, E ACHADOS HISTOPATOLÓGICOS DE BIÓPSIA DE ENDOMÉTRIO}

Barbosa CP, Camargo CS, Vince FAH

ejncam@uol.com.br

Introdução: A histeroscopia é padrão ouro para investigar o endométrio. $\mathrm{O}$ ultra-som transvaginal (USTV) apenas avalia espessura endometrial.
Dentre as indicações mais comuns de histeroscopia está o sangramento uterino pós-menopausa. Objetivo: Este estudo visa avaliar os resultados histeroscópicos em pacientes com sangramento uterino pós-menopausa. Métodos: Foram incluídas 113 pacientes com sangramento uterino pósmenopausa, divididas em: uso de terapia de reposição hormonal (TRH), espessura endometrial por USTV, resultados anátomo-patológicos e histeroscópicos. Resultados: Das 113 pacientes, não realizamos o procedimento em 15 por dificuldades técnicas. Das 98 restantes, $70(71 \%)$ não usavam TRH e 28 (29\%) usavam. Nas não usuárias $46(66 \%)$ apresentaram endométrio espessado $\left({ }^{3} 5 \mathrm{~mm}\right)$, o que ocorreu em $7(25 \%)$ das usuárias $\left({ }^{3} 10 \mathrm{~mm}\right)$. No diagnóstico histeroscópico, o endométrio apresentou-se alterado em $60 \%$ das pacientes sem TRH e em $40 \%$ daquelas com TRH. Dentre as alterações, a mais comum foi pólipo endometrial: $33(47 \%)$ pacientes sem TRH e $5(18 \%)$ com TRH. Em relação ao diagnóstico anátomo-patológico, $55 \%$ das pacientes não usuárias apresentaram alterações endometriais o que ocorreu em $26 \%$ das pacientes usuárias. O diagnóstico mais observado foi também pólipo endometrial: 8 (26\%) nas não usuárias e 3 (20\%) nas usuárias. Destacam-se 2 casos de carcinoma espino celular, 1 neoplasia maligna indiferenciada e 3 adenocarcinomas endometrióides, correspondendo a $6 \%$ de neoplasias. Conclusão: Concluímos que afecções intracavitárias são mais freqüentes em mulheres sem TRH, sendo a patologia mais comum o pólipo endometrial. Observamos que lesões malignas foram mais freqüentes nas não usuárias de TRH. Observou-se também que em 9 casos o material enviado para análise anátomo-patológica foi insuficiente para diagnóstico. Este fato reforça a importância da visão histeroscópica.

\section{DIFERENÇAS ENTRE A FREQÜÊNCIA DE REALIZAÇÃO DO EXAME DE PAPANICOLAOU ENTRE UMA POPULAÇÃO ATENDIDA PELO PROGRAMA DE AGENTES COMUNITÁRIOS DE SAÚdE E OUTRA NÃO ATENDIDA PELO MESMO}

Campos AS, El-Afiouni V, Hatakeyama TT, Marini F, Nazato DM, Pinto CMF

debnazato@ig.com.br

Introdução: Este trabalho tem o propósito de avaliar a atuação dos agentes comunitários de Saúde (ACS) frente à conscientização da necessidade da realização do exame de Papanicolaou (PPN). Casuística: 225 mulheres de região atendida pelo PCS e de 78 mulheres da região não atendida pelo serviço, dentro da faixa etária de 15 a 85 anos. Métodos: Questionário aplicado em duas populações diferentes, próximas uma da outra, no município de Santo André - São Paulo, no período de abril a setembro de 2002, atendida pelo programa de Agentes Comunitários de Saúde (PACS) e outra não atendida pelo mesmo serviço. Inclui dados econômicos e demográficos, além daqueles relativos à atividade sexual, uso de contraceptivos, auto-exame de mama e realização do exame de PPN. Conclusão: Há associação significativa entre início da atividade sexual e realização de PPN nas duas populações. Os resultados também mostram que $168(74,7 \%)$ mulheres atendidas pelo PACS e $63(80,8 \%)$ das não atendidas realizam o exame $(\mathrm{p}=0,12)$, verificando-se não haver diferença significativa entre ser atendido pelo PACS e a realização do exame. Porém, verificam-se diferenças significativas de renda entre os dois grupos $(p<0,001)$ e associação entre renda e realização de PPN $(p<0,001)$. No grupo PACS observa-se maior realização do exame de PPN em mulheres com renda inferior ou igual a $\mathrm{R} \$ 200,00$ em relação à população de mesma renda que não é atendida pelo mesmo serviço. Este fato ressalta o importante papel do PACS na conscientização sobre a realização do exame.

\section{EPIDEMIOLOGIA DO INFARTO AGUDO DO MIOCÁRDIO (IAM) NA CIDADE DE SANTO ANDRÉ NO ANO DE 2000}

Akerman M, Braga ALF, Cammarosano RAFA, Diaz TF, Gasparotti E, Martins LC

rafacammarosano@uol.com.br

Introdução: No Brasil as doenças do aparelho circulatório constituem a principal causa de mortalidade proporcional. Nesse grupo o IAM é indicado 
para desenvolvimento de padrões de qualidade, considerando-se o impacto na mortalidade, volume de internações que acarreta e sensibilidade a tecnologias hospitalares. Objetivo: Esse trabalho pretende verificar a incidência do IAM na cidade de Santo André. Métodos: Foram coletadas informações sobre internações hospitalares no Sistema Único de Saúde (SUS) por IAM em Santo André no ano de 200.Foi feita a análise descritiva de todas variáveis do estudo para o grupo todo e dividida por gênero. Foram utilizados os testes U de Mann-Whitney e Qui-Quadrado. Resultados: A média de idade do grupo todo foi 58 anos, o que não difere entre os gêneros. Observamos que a menor idade atingida entre os homens foi 24 anos e entre mulheres 25 anos. Houve um maior número de casos entre o sexo masculino $(67,8 \%)$ e entre os meses de novembro e janeiro. O dia da semana com o maior número de internações é sexta-feira (17\%), e o menor é sábado (9\%). Conclusão: O Brasil apresenta um quadro consolidado, porém dinâmico, de padrão de morbimortalidade pelo IAM com importantes características: altas taxas entre jovens e mulheres, e esse padrão se mantém em Santo André. A tendência mundial de diminuição das faixas etárias atingidas também se confirma. Com base nesse estudo e na literatura, observamos que os casos de IAM em Santo André tendem a seguir o mesmo rumo de outras cidades.

\section{ESTUDO EPIDEMIOLÓGICO DAS FRATURAS E LUXAÇÕES INSTÁVEIS DO ANEL PÉLVICO}

Fujiki E, Gonçalves FR, Mendonça RR, Pavan A, Saab L, Yamaguchi EM

lucianasaab@yahoo.com.br

Introdução: Tem havido um aumento na ocorrência das fraturas-luxações instáveis do anel pélvico. Daí a importância de se estudar a epidemiologia dessas fraturas. Métodos: 23 pacientes foram submetidos ao tratamento cirúrgico por fraturas e luxações da pelve. Foi utilizada a classificação Tile para fraturas do anel pélvico. A idade dos pacientes variou entre 18 e 53 anos. O tratamento constitui na redução cruenta e osteossíntese interna ou uso de fixadores externos para estabilizar fraturas. Casuística: O atropelamento foi a principal causa de fratura, seguido pelo acidente automobilístico. O sexo masculino foi predominante. As fraturas classificadas como tipo $\mathrm{C}$ foram as mais comuns. Dez fraturas foram classificadas como tipo B. De nove pacientes, atendidos nas primeiras 24 horas, três estavam hemodinamicamente instáveis, sendo estabilizados com fixador externo. Os 14 pacientes restantes vieram de outros serviços onde receberam os primeiros socorros; 5 pacientes apresentaram lesões de órgãos abdominais; 11 pacientes apresentaram fraturas associadas; 3 pacientes tiveram TCE. O fixador externo foi usado como tratamento definitivo em 4 pacientes. Em 17 casos, utilizou-se a osteossíntese interna. Conclusão: De acordo com o levantamento, podemos inferir que o sexo masculino, entre a terceira e quarta décadas, é o grupo mais atingido, sendo os atropelamentos e os acidentes automobilísticos os maiores fatores etiológicos. O uso de fixador externo temporariamente é útil nas emergências, e pode ser utilizado como tratamento definitivo.

\section{FRATURAS DO FÊMUR PROXIMAL NA INFÂNCIA}

\section{Dobashi ET, Hiratsuka J, Ksyvicks L, Milani C, Sá MMS, Sanjar FA}

ksyvickslivia@hotmail.com

Introdução: Fraturas do fêmur proximal são raras, correspondendo menos de $1 \%$ de todas lesões ósseas do esqueleto imaturo. Objetivo: 13 crianças portadoras de fraturas do fêmur proximal, sendo 6 do sexo masculino e 7 do feminino. Resultados: A média das idades foi 8,4 anos (de 6 meses a 14 anos). O lado direito foi acometido em 11 quadris. O tempo de seguimento variou de 1,5 anos até 12 anos (média 4,5 anos). Utilizamos classificação de DELBET (1907) e COLONA (1929), sendo 1 fratura $(7,7 \%)$ classificada como tipo I, $10(76,9 \%)$ tipo II, $1(7,7 \%)$ tipo III e 1 $(7,7 \%)$ tipo IV. De acordo com critérios de BOITZY (1971) o desvio entre fragmentos fraturados foi inferior a $50 \%$ em $9(69,2 \%)$, superior a $50 \%$ em $3(23,1 \%)$ e completa em $1(7,7 \%)$ paciente. Quanto ao mecanismo de trauma, $11(84,6 \%)$ foram submetidos aos acidentes envolvendo alta energia cinética (atropelamentos, quedas de altura) e $2(15,4 \%)$ de baixa energia (queda precedida por crise convulsiva). Nove $(69,2 \%)$ quadris foram tratados cirurgicamente (redução, osteossíntese, gesso pelvipodálico) e $4(30,8 \%)$ pelo método não operatório (redução, gesso pelvipodálico). Como complicações foram observadas: $3(23,1 \%)$ pseudoartroses, 3 $(23,1 \%)$ coxas varas, $1(7,5 \%)$ necrose avascular tipo I de RATLIFF (1962) e 1 (7,5\%)"anisomelia. Conclusão: A avaliação dos resultados funcionais classificou com bons $7(53,8 \%)$ quadris (sendo 6 submetidos ao tratamento cirúrgico imediato); $2(15,4 \%)$ regulares e $4(30,8 \%)$ maus resultados, segundo critérios de RATLIFF (1978) modificados por TUCCI NETO (1997).

\section{HELICOBACTER PYLORI NOGRANDE ABC PAULISTA - ASPECTOS CLÍNICOS E EPIDEMIOLÓGICOS EM 977 PACIENTES}

Blandi FA, Burdelis REM, Chehter EZ, Maximiano FL, Nogueira LFF, Xavier MFF

leoffnog@ hotmail.com

Introdução: No Brasil, existem poucos estudos que avaliem a incidência do Helicobacter pylori, assim como na região do Grande ABC Paulista. Esta se destaca por bom nível de desenvolvimento urbano (0.813) e renda per capita - U\$ 3250.00/ anuais. Procuramos: 1. avaliar a prevalência da infecção do Helicobacter pylori, 2. detectar manifestações dispéticas, 3. associar fatores de risco / nível sócio-econômico. Casuística: Incluímos 977 pacientes, $67 \%$ feminino, idade média 50,5 anos \pm 17,3 (18-95 anos), 53\% do estado de São Paulo, casados (58\%) e brancos 62\%. Destacouse azia em $45 \%$. Nos fatores de risco: o uso de antiinflamatórios não esteróides (AINEs) em $50 \%$, poucos fumantes $-20 \%$ e etilistas $-20 \%$. Avaliação sócio-econômica revelou: casa própria em $76 \%$ (média 3-4 cômodos - 53\%), saneamento básico em 95\%, mas escolaridade baixa $47 \%-1^{\circ}$ grau incompleto. Sorologia IgG para H. pylori foi positiva em 73\%. Métodos: Estudo aberto, seqüencial, prospectivo; realizado na Faculdade de Medicina do ABC, entre janeiro/2001 - março/2003. incluímos pacientes que, por qualquer razão, coletariam sangue (Anexo II), adultos, e responderam questionário sobre dispepsia / fatores de risco para doença péptica / avaliação sócio econômica. Submetemos uma amostra de sangue para pesquisa de Helicobacter pylori ( $\operatorname{IgG} / \operatorname{IgM}$ ). Conclusão: Apesar da população estudada ter bom nível sócio-econômico, verificouse elevada incidência de $H$. pylori. Clinicamente observamos muitos pacientes sintomáticos, especialmente com azia, provavelmente relacionada ao extensivo uso de AINES.

\section{INCIDÊNCIA DE LESÃO DA AORTA ABDOMINAL: BREVE REVISÃO DA LITERATURA}

Barsella AR, Benavides M, Guimarães SO, Polimanti AC, Silva KA, Velha LP

augustorafael@connectmed.com.br

Introdução: As lesões vasculares ocorrem em 6 a $12 \%$ dos casos, com mortalidade de 30 a $80 \%$. A aorta é acometida em cerca de 15 a $25 \%$ dos traumas vasculares abdominais, sendo que $80 \%$ dos pacientes apresentamse em choque. Há lesão em 3 ou mais órgãos em aproximadamente $30 \%$ dos casos e outra lesão vascular em 58\% destes. O comprometimento da aorta abdominal é de suma importância, já que o trauma neste segmento possui incidência de $25 \%$ dos casos acima do tronco celíaco e outros $25 \%$ acometendo a região do tronco celíaco e artéria renal; e 50\% no segmento da aorta infra-renal. Além disso, as taxas de mortalidade destas lesões são de $70 \%$ e $80 \%$, e $47 \%$; respectivamente. Objetivo: Demonstrar a incidência de lesão na aorta abdominal e sua importância com as taxas de sobrevida. Métodos: Realizamos busca e revisão na literatura sobre o tema, correlacionando cada artigo com a casuística e metodologia apresentada. Resultados: Os resultados apresentados nos permitem a elaboração deste pôster com a elaboração de um perfil das lesões da aorta abdominal com a correlação das taxas de mortalidade e sobrevida. Conclusão: Conclui-se que as lesões da artéria aorta abdominal são extremamente graves, necessitando de abordagens rápidas e precisas, além disso, a cinemática do trauma e o mecanismo de lesão podem sugerir um perfil desta lesão em sua localização. 


\section{INCIDÊNCIA DA LESÃO DA VEIA CAVA: BREVE REVISÃO DA LITERATURA}

Barsella AR, Benavides M, Guimarães SO, Polimanti AC, Silva KA, Velha LP

augustorafael@connectmed.com.br

Introdução: As lesões vasculares ocorrem em 6 a 12\% dos casos, com mortalidade de 30 a $80 \%$. A aorta é acometida em cerca de 15 a $25 \%$ dos traumas vasculares abdominais, sendo que $80 \%$ dos pacientes apresentamse em choque. O comprometimento da veia cava é de suma importância, já que o trauma neste segmento faz com que as vítimas cheguem aos hospitais hipotensas, hipotérmicas e com graves sinais de hipovolemia. Objetivo: Demonstrar a incidência de lesão na veia cava e sua importância com as taxas de sobrevida. Métodos: Realizamos busca e revisão na literatura sobre o tema, correlacionando cada artigo com a casuística e metodologia apresentada. Resultados: Os resultados apresentados nos permitem a elaboração deste pôster com a elaboração de um perfil das lesões da veia cava com a correlação das taxas de mortalidade e sobrevida. Conclusão: Conclui-se que as lesões da veia cava são extremamente graves, necessitando de abordagens rápidas e precisas, além disso, a cinemática do trauma e o mecanismo de lesão podem sugerir um perfil desta lesão em sua localização.

149. PERFIL BIO SÓCIO E ECONÔMICO DE 50 MULHERES ATENDIDAS NO GRUPO DE ATENÇÃO À MULHER - GRAM DA FACULDADE DE MEDICINA DA FUNDAÇÃO DO ABC

Fillipini R, Lara SG, Loddi S, Pacheco GA

si.loddi@bol.com.br

Introdução: Das neoplasias malignas da mulher, o câncer cérvico-uterino é o segundo mais importante, entretanto, atualmente a cobertura de exames preventivos se mantém com baixos índices apesar da OMS estabelecer em $85 \%$ de cobertura mínima para impacto epidemiológico. Como finalidade de prevenir agravos à saúde da mulher, o Grupo de Atenção à Mulher desenvolve atividades interdisciplinares, Enfermagem e Medicina da FMABC, à mulher nos níveis primário e secundário, com ações educativas, consulta de enfermagem, Papanicolaou e Teste Schiller e encaminhamento para as especialidades médicas. Objetivo: Caracterizar o perfil de mulheres atendidas no GRAM e identificar fatores que possibilitem estabelecer às ações de saúde com mais eficácia e resolutividade. Métodos: Polução de estudo com 50 mulheres, variáveis biológicas e sociais; dados obtidos e analisados pelo EPINFO em tabelas com números percentuais e absolutos; nível de significância p<0,05. Resultados: Predomínio: 59,2\% mulheres mais de 40 anos e 14,4\% menos de 30; situação marital: $60 \%$ casada ou amasiada; Indicadores Sociais: $52 \%$ com ensino fundamental incompleto; famílias com 2 ou menos salários mínimos mês $(46,9 \%)$. Consideram-se fatores positivos as condições de moradia (92\% alvenaria) e recursos públicos presentes (96\%); quanto mais envelhecentes menos mantém atividade sexual (valor $\mathrm{p}<0,03$ ). Conclusão: Educação sexual para prevenção do colo uterino em mulheres jovens; estabelecer ações de educação em saúde nas fases reprodutiva, climatério e menopausa que garantam a aderência ao programa.

150. PERFIL DOS PACIENTES PORTADORES DE DIABETES MELLITUS ATENDIDOS NO AMBULATÓRIO DE ENDOCRINOLOGIA DA FACULDADE DE MEDICINA DO ABC

Akerman M, Fernandes APC, Kassab C, Mendonça RR, Pitta TS, Rodrigues PFB

rafaelamendonca@ig.com.br

Introdução: O Diabetes Mellitus tem incidência significativa, crescente em todo o mundo e elevado custo sócio-econômico. No Brasil, a taxa de prevalência é 7,60\% na população adulta (30-69 anos). Para se desenvolver projetos de prevenção são necessários estudos mostrando o perfil dos diabéticos. Objetivo: Apresentar o perfil dos diabéticos atendidos no ambulatório de endocrinologia da Faculdade de Medicina do ABC. Métodos: Estudo retrospectivo que analisou 88 prontuários entre julho e outubro de 2001. Dos 34 diabéticos encontrados, analisou-se: sexo, idade, raça, tabagismo, co-morbidades, atividade física, antecedentes familiares, tipo de tratamento e IMC (índice de massa corpórea). Resultados: Dezenove mulheres e quinze homens, com idade média de 59 anos. Dois negros, dezesseis brancos, cinco pardos; onze dados não constavam. Oito eram tabagistas, oito não tabagistas, nove pararam de fumar e não há dados de nove. Quatro pacientes realizavam atividade física e onze eram sedentários; dados de dezenove não constavam. Onze tinham parentes com Diabetes Mellitus, cinco não, oito não sabiam; de dez falta informação. Familiares mais afetados: mãe e irmão. Seis tinham IMC normal, nove sobrepeso, oito obesos e onze sem informação. Onze pacientes usavam hipoglicemiantes orais e dez insulina. Quatro faziam apenas tratamento dietético. Três associavam insulina e hipoglicemiantes orais e faltam seis dados. Trinta e dois pacientes tinham co-morbidades, sendo estas principalmente dislipidemias, HAS e alterações cardiovasculares. Conclusão: Os pacientes são, em sua maioria, brancos, sedentários, apresentam sobrepeso e idade média de 59 anos. Estudos posteriores são necessários para melhor análise do perfil dos diabéticos e para isso precisa-se do correto preenchimento dos prontuários, o que não foi observado.

\section{PERFIL DO=S PARTICIPANTES DA CAMPANHA DE DIABE- TES REALIZADA EM SANTO ANDRÉ EM 2002}

Camargo CS, Kassab C, Kassab P, Mendonça RR, Reis A, Rodrigues PFB

rafaelamendonca@hotmail.com

Introdução: O Diabetes Mellitus é uma doença cuja prevalência e incidência vem crescendo nos últimos anos.É fator de risco para doenças cardiovasculares. Segundo a OMS atualmente há 150 milhões de portadores de Diabetes no mundo. Isso tem impacto significativo na Saúde Pública, consumindo cerca de $20 \%$ dos recursos governamentais destinados à saúde. Objetivo: Avaliar o perfil das pessoas atendidas na Campanha de diabetes realizada no município de santo André - SP em 2002 e verificar o conhecimento da população sobre ser ou não portadora da doença. Métodos: Realizado um estudo transversal com amostragem casual simples da população de Santo André. Responderam a um questionário 627 pessoas. Itens analisados: sexo, idade, portador de Diabetes, tempo de doença, familiares diabéticos e período de jejum,; subseqüentemente se mediu a glicemia capilar, considerando-se normais valores inferiores a $160 \mathrm{mg} / \mathrm{dL}$. Resultados: Compareceram à Campanha 376 mulheres e 251 homens com idade entre 41 e 70 anos. A maioria (502) desconhecia ser ou não portadora da doença. Além disso, 327 tinham familiares acometidos e 558 não estavam em jejum absoluto. A glicemia capilar foi menor que $110 \mathrm{mg} / \mathrm{dL}$ em 436 pessoas $(69 \%)$ e maior que $160 \mathrm{mg} / \mathrm{dL}$ em $55(8,7 \%)$ sendo que destas $73 \%$ já sabia ser diabética. Conclusão: A população analisada constituiu-se de mulheres entre $4^{\mathrm{a}}$ e $7^{\mathrm{a}}$ década de vida e a maioria das pessoas não tinha informação sobre ser portadora da doença.

\section{PERFIL EPIDEMIOLÓGICO DA POLIDACTILIA E RESULTADOS COM O TRATAMENTO CIRÚRGICO}

Aita MA, Barella SM, Fukushima WY, Mendonça RR, Mitev AG, Tardini R simaluf32@yahoo.com.br

Introdução: A polidactilia é uma má formação do tipo duplicação dos dedos, que pode ser radial, central ou ulnar. A polidactilia radial é estudada como duplicação do polegar. A polidactilia central é estudada como polisindactilia, freqüentemente é bilateral e pode ser do $2^{\circ}, 3^{\circ}$ e $4^{\circ}$ dedo, sendo o mais comum o médio. Objetivo: Apresentar o perfil epidemiológico da polidactilia nas mãos, os tipos, classificações e resultados dos tratamentos cirúrgicos realizados. Métodos: Na Faculdade de Medicina do ABC e Hospital Ipiranga, 24 pacientes foram estudados de janeiro de 2001 a maio de 2003 e submetidos à ressecção do dedo extranumerário. Resultados: Treze pacientes do sexo masculino e onze do feminino, com idade entre oito meses e 23 anos. Quinze pacientes com duplicação de polegar, classificados, segundo Wassel, sendo cinco do tipo II e dez do tipo IV. Os polegares do tipo II foram submetidos à ressecção do broto menor e menos funcional. Nos casos tipo IV, três foram submetidos à ressecção do dedo da extremidade ulnar. Os nove pacientes com polidactilia ulnar foram submetidos à ressecção simples do broto. Quanto ao lado acometido, do tipo ulnar foram sete do lado direito e dois do esquerdo; do tipo radial foram dois do lado direito, seis do esquerdo e sete bilaterais. Quanto ao sexo, no grupo do tipo pós-axial, sete eram masculinos e dois 
femininos; no tipo pré-axial, seis eram masculinos e nove eram femininos. Não houve acometimento central. Conclusão: Os resultados estéticos e funcionais foram satisfatórios. O polegar foi o dedo mais acometido (principalmente do tipo IV).

153. PREVENÇÃO DE FATORES DE RISCO PARA ATEROSCLEROSE E VARIÁVEIS NUTRICIONAIS EM ESTUDANTES DE MEDICINA DO ESTADO DE SÃO PAULO

Alves CR, Bertolami A, Bricarello LP, Camargo CS, Fernandes J, Hiratsuka J adribertolami@uol.com.br

Introdução: A doença cardiovascular aterosclerótica é considerada problema de saúde pública no Brasil e no mundo. Sabe-se que determinados fatores de risco propiciam a instalação deste quadro. Objetivo: Conhecer a prevalência de fatores de risco para aterosclerose e sua relação com antecedentes pessoais e estilo de vida em uma população jovem. Métodos: Foram entrevistados 182 estudantes de medicina com idades entre 19 e 30 anos, durante o ano de 2002. Utilizou-se um questionário que inclui antecedentes pessoais, hábitos de vida e freqüência alimentar. Resultados: Em relação aos fatores de risco investigados, foi diagnosticado, segundo os critérios adotados, que $12 \%$ da população apresenta pelo menos um dos fatores de risco para aterosclerose (Tabela). Com relação aos hábitos alimentares diários dos entrevistados, verificou-se por meio de freqüência alimentar que $63 \%$ consomem verduras, $54 \%$ legumes, $86 \%$ frutas e sucos naturais, $34 \%$ adoçantes e $21 \%$ refrigerantes dietéticos e $34 \%$ fazem uso do leite desnatado. Constatou-se que $60 \%$ pratica algum tipo de atividade física regularmente.

\begin{tabular}{|l|l|}
\hline Fatores de risco & $\mathbf{\%}$ \\
\hline Diabetes Mellitus & 2 \\
\hline Hipercolesterolemia & 5 \\
\hline Hipertrigliceridemia & 2 \\
\hline Dislipidemia Mista & 2 \\
\hline Hipertensão & 1 \\
\hline Tabagismo & 7 \\
\hline
\end{tabular}

Conclusão: Verificou-se baixa prevalência dos fatores de risco avaliados, provavelmente pela faixa etária mais jovem da amostra. Os hábitos alimentares da população apresentam-se adequados nos quesitos demonstrados acima, sugerindo preocupação com a qualidade de vida e com a boa forma física, já que somente $10 \%$ dos entrevistados encontram-se na faixa do sobrepeso ou obesidade, e que a maior parte investigada pratica atividades físicas regulares e não fumam.

154. TRANSTORNO DO SONO: ESTUDO DA QUALIDADE DE SONO DE UMA POPULAÇÃO DE PRIMEIROS ANISTAS DE MEDICINA

D’elia G, Lima NCP, Oliveira LS, Sadatsune DE, Sadatsune EJ, Zuliani LMM

luiza_o@hotmail.com

Introdução: A saúde do aluno de medicina é objeto de estudo de crescente preocupação na literatura médica. Vários estudos mostram uma incidência aumentada de depressão e ansiedade nessa população. O sono é um dos principais fatores associados à qualidade de vida e fonte de estresse, sobre os quais as exigências do curso médico tem alto impacto. Objetivo: Este estudo procura avaliar a qualidade do sono e seu impacto na sonolência diurna do primeiro anista do curso de Medicina da FMABC. Métodos: Foram aplicados questionários de auto-preenchimennto, dando enfoque à qualidade de sono dessa população. Resultados: Foram estudadas as respostas de 93 questionários. Esta população é composta de 32,22\% homens e $67,78 \%$ mulheres, com idade média $19,97 \% \pm 1,11$, a qual relata dormir em média 6,67 $\pm 1,19$ horas por noite. Quanto à qualidade do sono, foi verificado sono agitado por $42,22 \%$ e cansaço ao acordar por $44,44 \%$ da amostra. Foi observada alta sonolência diurna, relatada como alta e moderada chance de dormir em aula à tarde (70\%). Conclusão: Os resultados observados sugerem baixa qualidade de sono nessa população, com possível impacto negativo no seu desempenho cotidiano. 